\title{
Heterogeneous photocatalysis of azides: extending nitrenes photochemistry to longer wavelengths
}

Received 00th January 20xx,

\author{
Ignacio D. Lemir ${ }^{a, b}$, Juan Argüello ${ }^{b}$, Anabel E. Lanterna*a, Juan C. Scaiano*a
}

DOI: $10.1039 / \times 0 \times x 00000 x$

The photodecomposition of azides to generate nitrenes usually requires wavelengths in the $<300 \mathrm{~nm}$ region. Here we show this reaction can be readily performed in the UVA region $(368 \mathrm{~nm})$ when catalyzed by $\mathrm{Pd}$-decorated $\mathrm{TiO}_{2}$. In aqueous media the reaction leads to amines, with water acting as $\mathrm{H}$ source; however, in nonprotic and non-nucleophilic media - such as acetonitrile - nitrenes recombine to yield azo compounds, while azirine-mediated trapping occurs in the presence of nucleophiles. The heterogeneous process facilitates catalyst separation while showing great chemoselectivity and high yields.

In this work, we demonstrate that the conversion of azides to nitrenes can be performed at long wavelengths (UVA) when Pddecorated- $\mathrm{TiO}_{2}\left(\mathrm{Pd} @ \mathrm{TiO}_{2}\right)$ is the catalyst, leading to formation of azo compounds, azirine mediated trapping, and reduction to amines, all easily controlled by experimental parameters. Aqueous media leads to water as the sole $\mathrm{H}$ source for the reduction of azides into anilines using $\mathrm{Pd}$-decorated $\mathrm{TiO}_{2}$ as photocatalyst. The reaction is carried out under mild conditions - water solvent, room temperature, inert atmosphere, UVA irradiation, heterogeneous catalyst - with great chemoselectivity and high yields. These mild reductive conditions usually lead to better selectivity and facilitate the reaction workup, as the catalyst can be easily separated by centrifugation or filtration, whereas oxidised solvent debris can be eliminated during solvent removal. In addition, the use of longer UV irradiation (UVA) minimises photodegradation of products, a common issue from photochemistry performed under UVB or UVC irradiation.

Department of Chemistry and Biomolecular Science and Centre for Advanced Materials Research (CAMaR), University of Ottawa, 10 Marie Curie, Ottawa, ON K1N 6N5, Canada.

b. INFIQC-CONICET-UNC, Departamento de Química Orgánica, Facultad de Ciencias Químicas, Universidad Nacion-al de Córdoba, Ciudad Universitaria, X500OHUA Córdoba, Argentina.

Electronic Supplementary Information (ESI) available: Details of synthesis and MS and NMR spectroscopy, photo-catalytic reduction, reusability tests, absorption spectra. See DOI: 10.1039/x0xx00000x
The reduction of azides to amines is a valuable synthetic approach due to the azide's ease of preparation and good stability (room temperature and air) and their important role as protecting groups. ${ }^{1,2}$ This process is frequently performed under reductive conditions using high $\mathrm{H}_{2}$ pressures $^{3-5}$ or hydride sources 6,7 that leave organic debris behind. ${ }^{8,9}$ The direct photolysis of aromatic azides could also yield the formation of amines by promoting the extrusion of nitrogen and generation of nitrenes; ${ }^{10,11}$ however, this well-known process is usually carried out under extreme irradiation conditions (<300 nm). Recent contributions ${ }^{12,13}$ have explored the use of visible light, although using non-green solvents and harsh $\mathrm{H}$ sources. The use of copper-based catalysts was also explored, taking advantage of the well-known interaction between $\mathrm{Cu}$ and azide groups. ${ }^{3,6}$, 14 Palladium species are also known as promoters for $\mathrm{N}_{2}$ extrusion from azides. ${ }^{15}$ We have previously shown that the use of metal-decorated $\mathrm{TiO}_{2}$ as photocatalyst can facilitate transfer hydrogenation reactions using the solvent as $\mathrm{H}$ source. ${ }^{16,17}$ Particularly, it has been suggested that $\mathrm{Pd}-\mathrm{H}$ species are prone to be generated in materials such as $\mathrm{Pd}$-decorated $\mathrm{TiO}_{2}$ $\left(\mathrm{Pd} @ \mathrm{TiO}_{2}\right)$ under illumination. ${ }^{16}$ These species can act as reductive sites for suitable acceptors on the Pd surface. Additionally, Pd@ $\mathrm{TiO}_{2}$ has shown great efficiency as a heterogeneous photocatalyst, capable of retaining catalytic activity upon several reusability cycles under different conditions. 18

Here, we used our previously reported Pd@TiO 2 16, 19 (see details in $\mathrm{SI}$ ) to reduce different organic azides in water under mild conditions obtaining yields up to $98 \%$. A series of control reactions (Table S1) were carried out to help us understand the system. As previously noted, the presence of Pd is critical to ensure optimal charge carrier transportation, and the concomitant delay of the $\mathrm{TiO}_{2}$ electron-hole recombination. ${ }^{20}$ Not surprisingly, the reaction does not work when the catalyst is replaced by bare $\mathrm{TiO}_{2}$. The reaction can proceed under UVA irradiation ( $368 \mathrm{~nm}$ LED) but no products are detected when visible light (465 $\mathrm{nm}$ LED) is used instead. This suggests that excitation of $\mathrm{TiO}_{2}$ is required for the reaction to proceed. The 
absorption spectra of the azide reagents show they can only absorb light at shorter wavelengths, below the emission region of the LED used for excitation (Figure S1). This shows that the aromatic amines are not obtained by direct photolysis of aromatic azides, but rather through a UVA light-assisted hydrogenation of azide on $\mathrm{Pd@TiO}$ (Scheme 1). We have previously shown that $\mathrm{Pd}$ decoration can extend the absorption profile of $\mathrm{TiO}_{2}$ into the visible region, but that the photocatalytic activity of the material can change greatly depending on the irradiation wavelength used. ${ }^{21}$ Here we propose that $\mathrm{Pd}$ can also facilitate $\mathrm{N}_{2}$ extrusion under longer UV light irradiation through azide $\pi$-Pd interactions (Scheme 2); whilst, on the other side of the oxidation cycle, water can donate an electron to the photogenerated hole, generating reactive oxygen species (e.g., $\left.\mathrm{HO}^{*}\right)^{22}$ and acting as $\mathrm{H}$ source $\left(\mathrm{H}^{+}\right)$. Noteworthy, the heterogeneous nature of the process was confirmed by leaching experiments, where the reaction is unable to proceed once the heterogeneous catalyst is filtered out of the solution (Figure 1, left). Additionally, the catalyst can be reused several times without loss of photocatalytic activity (Figure 1, right).

A- Traditional photochemistry: middle UV

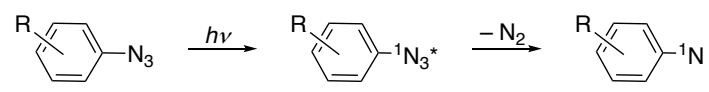

B- Semiconductor Photocatalysis: near UV - this work

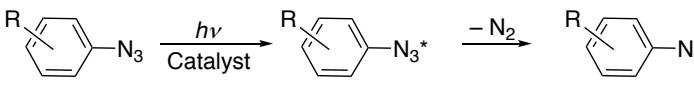

Scheme 1. Photochemistry of azides: formation of nitrene intermediates.

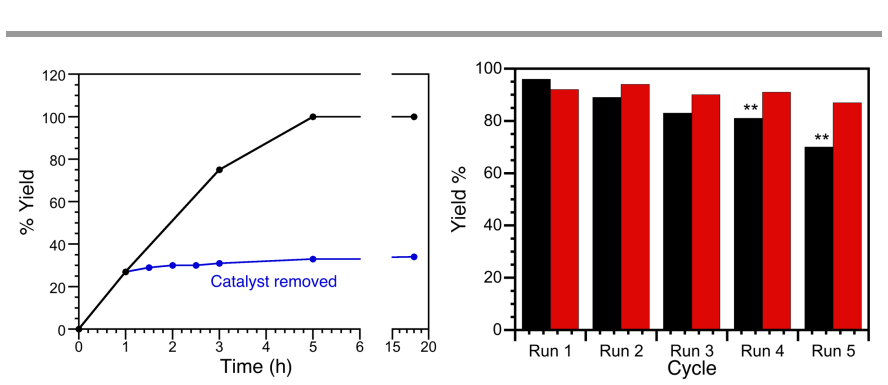

Figure 1. Left: Leaching test: reaction kinetics followed in the presence of catalyst (black) or removing the catalyst after $1 \mathrm{~h}$ (blue). Right: reusability of the catalyst is assessed after separation and reuse of catalyst (black) or without separation by subsequent additions of the reagent (red). The double stars $(* *)$ indicate only 6 out of $8 \mathrm{mg}$ of catalyst were recovered and reused for these reactions.

Based on the control reactions carried shown in table S1, we propose the mechanism of amine formation upon UVA excitation in aqueous media shown in Scheme 2. Since both Pd and UV light (excitation of $\mathrm{TiO}_{2}$ ) are necessary for the reaction to occur, we believe the excited electrons in the conduction band of $\mathrm{TiO}_{2}$ can be trapped by PdNPs to reduce $\mathrm{H}^{+}$and form hydride species on the Pd surface (Pd-H). Concomitantly, azides can undergo $\mathrm{N}_{2}$ extrusion (plausibly assisted by $\boldsymbol{\pi}$-Pd interactions in the cases of aromatic azides)-to produce nitrenes that can be stabilized on the Pd surface in a manner similar to the documented interaction of nitrogen centred species with $\mathrm{PdNP}{ }^{23}$ and $\mathrm{Pd}$ complexes. ${ }^{15}$ At this point, with $\mathrm{N}_{2}$ gone, the fate of the nitrenes will be determined by their interactions with available hydrogen atoms or other organic materials that can populate the Pd surface. The discussion that follows centres on selected examples from the scope of the reaction that is presented later in this contribution.

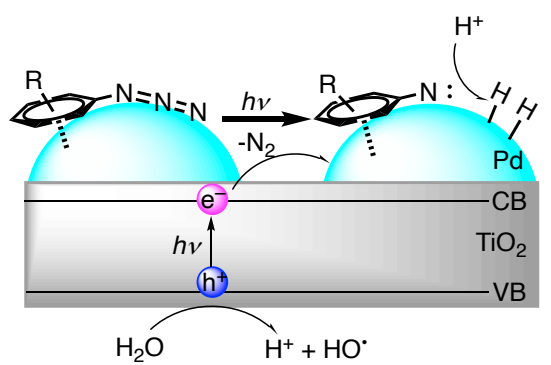

Scheme 2. Proposed $\pi$-Pd interactions-assisted mechanism for the photocatalytic formation of anilines via nitrene intermediate.

Using water as solvent, the presence of hydrides on the Pd surface - following band gap excitation of the titania support and electron migration to the PdNP - readily leads to the reduction of the nitrene to the corresponding amine. A comparison of phenyl azide ( $92 \%$ yield) with octyl azide ( $28 \%$ yield) serves to illustrate the importance of $\boldsymbol{\pi}$ interactions as part of the process that brings the substrate to the catalytically active sites. When the reaction is carried out in $D_{2} O$, the deuterated product is detected by NMR, ${ }^{24}$ supporting water as $\mathrm{H} / \mathrm{D}$ source (Figures S2-S3). When the solvent is acetonitrile, the surface generation of nitrene seems to occur just as efficiently as in water, but hydride formation on the Pd surface (or the release of hydride) seems to be far less efficient, and surface nitrenes dimerize yielding azobenzene (68\% isolated yield) as the only detectable product, as indicated in Scheme 3 below, where square brackets designate surface processes. Furthermore, we wondered whether amine formation in water could be mediated by the azo compound. To this effect we subjected azobenzene to the aqueous illumination protocol used for the azide, and found the corresponding hydrazine as the only product, while no aniline was detected; thus, confirming that azobenzene is not in the reaction path leading to aniline.

$$
\begin{aligned}
& \left.\mathrm{ArN}_{3} \stackrel{[]}{\longrightarrow}\left[\mathrm{ArN}_{3}\right] \stackrel{\mathrm{hv}}{\longrightarrow}[\mathrm{ArN}:] \stackrel{\mathrm{H}_{2} \mathrm{O},(\mathrm{H})}{\mathrm{AcN}}[\mathrm{ArN}=\mathrm{NArNH}] \longrightarrow \mathrm{ArNH}_{2}\right] \longrightarrow \mathrm{ArN}=\mathrm{NAr} \\
& \text { }
\end{aligned}
$$

Scheme 3. Proposed mechanisms for the reaction of nitrenes in water versus acetonitrile $(\mathrm{ACN})$ solvents. [ ] represent surface processes. Catalyst not shown for simplicity.

In the case of nitrenes in solution, singlets and triplets show distinct characteristic behaviour. ${ }^{11}$ We note that in the schemes and in the discussion above we have not mentioned multiplicity; we assume that in the presence of the palladium surface ST interconversion will be fast and perhaps these species are largely indistinguishable when located on the metal surface. Additionally, given the geometric opportunity, surface nitrenes can undergo intramolecular processes, as indicated in the carbazole formation (Scheme $4 \mathrm{~A}$ ), taking place in water with 
$>75 \%$ isolated yield. Further, the reaction shows typical aziridine chemistry (Scheme 4B). It is unclear at this point whether aziridines are in equilibrium with surface nitrenes, or whether the reaction is driven by the addition of $\mathrm{X}-\mathrm{H}$. In any event, there is literature precedent for equilibration between nitrenes and azirines (Scheme S2). ${ }^{11}$ When the reaction is performed in the presence of diisopropylamine (DIPA), the corresponding ortho-substituted product is obtained quantitatively. Moreover, when the azide incorporates a carbonyl moiety the product can be readily explained by the Curtius rearrangement (Scheme 5, table 1, entry 20) to produce only isocyanate, a typical product of nitrene rearrangement. ${ }^{25}$ Notably, no hydrogenation product (amide formation) is found, suggesting concerted Curtius rearrangement without formation of benzoyl nitrene intermediate. Additionally, the formation of aniline via hydrolysis was not detected, probably due to the neutrality conditions of the reaction $(\mathrm{pH} \sim$ neutral).

A-Intramolecular cyclization: formation of carbazole

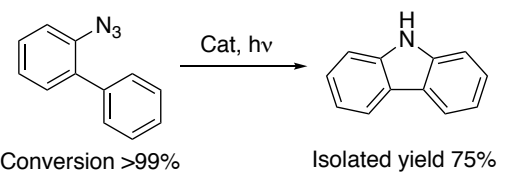

B-Aziridine nucleophilic substitution

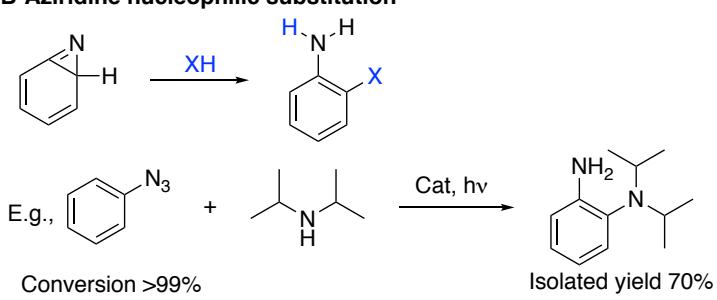

Scheme 4. A- Formation of carbazole, B- Nucleophilic substitution of aziridines.

The reduction of aryl azides (Table 1 ) shows some dependence on the electronic nature of the substituent group. Thus, aryl azides substituted with electron-donating groups (EDG) in para position show slightly higher reaction yields (80-97\%) comparing to those obtained with electron-withdrawing groups (EWG) in the same position (50-80\%), whereas -with the exception of $\mathrm{F}-$ no reaction was detected for halogen substituents, likely due to heavy atom effect (i.e., intersystem crossing generating triplet states favoured in the presence of heavy atoms). ${ }^{26,27}$ Notably, the reaction shows $100 \%$ selectivity towards reduction of azide group versus ketone (entry 8), carboxyl (entry 9), nitro (entry 10), and alkene groups (entry 13). These results further support our proposed mechanism. Particularly, the absence of cyclization in the alkene derivative (entry 13) together with the lack of reactivity of halogenated compounds do not support an electron transfer pathway, where the iodine and bromine derivatives are expected to undergo dehalogenation and chlorine derivates to form $p$ chloroaniline. ${ }^{28}$ Additionally, biphenyl azides (entries 14-15) can undergo intramolecular cyclization to furnish carbazole products with high yields and selectivity. Moreover, the reaction of benzyl azide yields the imine product with $100 \%$ selectivity (entry 18). This species is often seen as one of the by- products in the synthesis of nitriles from azides, ${ }^{29}$ and can be favoured after tuning reaction conditions. ${ }^{30}$ Further, under the same reaction conditions lower yields of aliphatic amines are expected as can be seen in the case of octyl amine $(28 \%$ isolated yield, entry 19).

In conclusion, we have demonstrated that the heterogeneous photocatalytic conversion of azides to nitrenes can be initiated

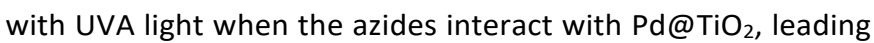
to surface-stabilized nitrenes; their fate depends on the reactive opportunities present on the surface and determined by the solvent and potential presence of nucleophiles. Specifically, reduction of organo-azides to amines can be achieved by using water as $\mathrm{H}$ source. A broad range of organo-azides can be reduced with excellent yields showing the reaction chemoselectivity towards azides versus all terminal alkene, nitro, carbonyl, and carboxylic groups. Furthermore, when the media is non-protic and non-nucleophilic, as is the case in acetonitrile, the surface nitrenes undergo recombination to produce the azo compound. In a nutshell, this strategy can be used to replace the conventional photolysis of azides, facilitating the chemistry of nitrenes at longer wavelengths, as azides do not usually absorb in the UVA region.

\section{Conflicts of interest}

There are no conflicts to declare.

\section{Acknowledgement}

This work was supported by the Natural Sciences and Engineering Research Council of Canada, the Canada Foundation for Innovation, the Canada Research Chairs Program and funding from Canada's International Development Research Centre (IDRC). IDL is a grateful recipient of the Emerging Leaders of the Americas Program (ELAP) scholarship. 
Table 1. Scope of the photocatalytic reduction of azides ${ }^{a}$

(10\%)

[a] Reaction conditions: $0.2 \mathrm{mmol}$ organic azide, $8 \mathrm{mg}$ of $\mathrm{Pd} @ \mathrm{TiO}_{2}, 2 \mathrm{ml}$ of $\mathrm{H}_{2} \mathrm{O}, \mathrm{A}$ atmosphere, UVA irradiation (368 nm LED working at $0.06 \mathrm{~W} / \mathrm{cm}^{2}$ ), $5 \mathrm{~h}$. [b] Conversions were estimated by GC-MS. [c]Purification was performed by flash column chromatography and the products characterized by $\mathrm{MS},{ }^{1} \mathrm{H}$ and ${ }^{13} \mathrm{C} N M R$. Values between brackets correspond to the selectivity towards reduction of azide group.

\section{References}

1. S. Pothukanuri and N. Winssinger, Org. Lett., 2007, 9, 22232225.
2. H. S. G. Beckmann and V. Wittmann, in Organic Azides, 2009, DOI: 10.1002/9780470682517.ch16, pp. 469-490.

3. S. Ahammed, A. Saha and B. C. Ranu, J. Org. Chem., 2011, 76, 7235-7239.

4. N. Arai, N. Onodera and T. Ohkuma, Tetrahedron Lett., 2016, 57, 4183-4186.

5. A. K. Rathi, M. B. Gawande, V. Ranc, J. Pechousek, M. Petr, K. Cepe, R. S. Varma and R. Zboril, Cat. Sci. Tech., 2016, 6, 152-160. 6. Y. Chen, L. X. Yang, X. Zhang, S. Q. Deng, L. You and Y. Liu, Chem. Select, 2018, 3, 96-99.

7. J. G. Du, G. Xu, H. K. Lin, G. W. Wang, M. L. Tao and W. Q. Zhang, Green Chem., 2016, 18, 2726-2735.

8. Y. P. Wu, B. Yang, J. Tian, S. B. Yu, H. Wang, D. W. Zhang, Y. Liu and Z. T. Li, Chem. Comm., 2017, 53, 13367-13370.

9. N. Chandna, F. Kaur, S. Kumar and N. Jain, Green Chem., 2017, 19, 4268-4271.

10. D. E. Falvey and A. D. Gudmundsdottir, Nitrenes and nitrenium ions, Wiley, Hoboken, New Jersey, 2013.

11. M. S. Platz, in Reactive Intermediate Chemistry, eds. M. Jones, Jr., R. A. Moss, M. Platz and M. Jones, Wiley-Interscience, Hoboken, N.J., 2004, ch. 11, pp. 501-560.

12. Y.-P. Wu, B. Yang, J. Tian, S.-B. Yu, H. Wang, D.-W. Zhang, Y. Liu and Z.-T. Li, Chem. Commun., 2017, 53, 13367-13370.

13. Y.-P. Wu, M. Yan, Z.-Z. Gao, J.-L. Hou, H. Wang, D.-W. Zhang, J. Zhang and Z.-T. Li, Chin. Chem. Lett., 2019, 30, 1383-1386.

14. B. Zelenay, M. Besora, Z. Monasterio, D. Ventura-Espinosa, A. J. P. White, F. Maseras and S. Diez-Gonzalez, Cat. Sci. Tech., 2018, 8, 5763-5773.

15. Z. Zhang, Z. Li, B. Fu and Z. Zhang, Chem. Comm., 2015, 51, $16312-16315$.

16. A. Elhage, A. E. Lanterna and J. C. Scaiano, ACS Catal., 2017, 7, 250-255.

17. B. Wang, K. Duke, J. C. Scaiano and A. E. Lanterna, J. Catal., 2019, 379, 33-38.

18. A. Elhage, A. E. Lanterna and J. C. Scaiano, Chem. Sci., 2019, 10, 1419-1425.

19. A. Hainer, N. Marina, S. Rincon, P. Costa, A. E. Lanterna and J. C. Scaiano, J. Am. Chem. Soc., 2019, 141, 4531-4535.

20. A. S. Hainer, J. S. Hodgins, V. Sandre, M. Vallieres, A. E. Lanterna and J. C. Scaiano, ACS Ener. Lett., 2018, 3, 542-545.

21. A. Elhage, A. E. Lanterna and J. C. Scaiano, ACS Sust. Chem. Eng., 2018, 6, 1717-1722.

22. M. Jonsson, in Visible-Light-Active Photocatalysis:

Nanostructured Catalyst Design, Mechanisms, and Applications, ed. S. Ghosh, Wiley-VCH, Weinhem, 2018.

23. S. Singha, M. Sahoo and K. M. Parida, Dalton Trans., 2011, 40, 7130-7132.

24. A. Gevorgyan, S. Mkrtchyan, T. Grigoryan and V. O. laroshenko, ChemPlusChem, 2018, 83, 375-382.

25. A. K. Ghosh, A. Sarkar and M. Brindisi, Org. Biomol. Chem., 2018, 16, 2006-2027.

26. D. V. Korchagin, A. V. Akimov, E. A. Yureva, S. M. Aldoshin and E. Y. Misochko, Chem. Phys. Lett., 2016, 659, 234-236.

27. N. P. Gritsan, D. Tigelaar and M. S. Platz, J. Phys. Chem. A, 1999, 103, 4465-4469.

28. Y. Y. Chen, A. S. Kamlet, J. B. Steinman and D. R. Liu, Nature Chem., 2011, 3, 146-153.

29. J. L. He, K. Yamaguchi and N. Mizuno, J. Org. Chem., 2011, 76 4606-4610.

30. L. Martinez-Sarti and S. Diez-Gonzalez, ChemCatChem, 2013, 5, 1722-1724. 